\title{
Delayed diagnosis of congenital thrombotic thrombocytopenic purpura in a patient with recurrent strokes
}

\author{
Marina Beltrami-Moreira ${ }^{1}$ - Maria T. DeSancho ${ }^{1}$ (D) \\ Accepted: 29 December 2021 / Published online: 12 January 2022 \\ (c) The Author(s), under exclusive licence to Springer Science+Business Media, LLC, part of Springer Nature 2022
}

\begin{abstract}
Congenital thrombotic thrombocytopenic purpura (cTTP) is caused by ADAMTS13 mutations and associated with high risk of microvascular thrombosis. A 58 year old female had an ischemic stroke during hormonal fertility, and a TIA a year after. She suffered another stroke 18 years later while on warfarin. Four months after she developed severe thrombocytopenia, mild anemia, and increased LDH. Blood film showed schistocytes. She was hospitalized with presumptive TTP. ADAMTS 13 activity was undetectable without inhibitor. She developed another stroke and received plasma exchange. A homozygote ADAMTS 13 mutation was identified. Despite plasma, the ADAMTS13 activity remained $<10 \%$ and she had another stroke. Recombinant ADAMTS13 therapy was obtained through compassionate use. She receives weekly infusions maintaining ADAMTS13 trough levels above $10 \%$ without thrombotic recurrences. This case underscores the need to recognize cTTP as a cause of cryptogenic strokes, and the diagnostic value of the peripheral blood film. rADAMTS13 replacement may prevent recurrences.
\end{abstract}

Keywords Congenital thrombotic thrombocytopenia purpura $\cdot$ Cryptogenic recurrent strokes

\section{Highlights}

- Congenital thrombotic thrombocytopenic purpura (cTTP) is a rare autosomal recessive disorder resulting from ADAMTS13 gene mutations.

- Patients with cTTP are at increased risk for manifestations of microvascular thrombosis such as recurrent strokes.

- Evaluation of the peripheral blood smear along with marked decreased ADAMTS13 activity and absent inhibitor is essential in leading to the diagnosis of cTTP.

- Management with recombinant ADAMTS 13 markedly improved the care of this patient.

This case report was presented in part as a poster abstract at the ISTH annual meeting in 2021.

Maria T. DeSancho

mtd2002@med.cornell.edu

1 Division of Hematology and Oncology, Department of Medicine, Weill Cornell Medicine/New York Presbyterian Hospital, 1305 York Avenue 7th Floor Room 51, New York, NY 10021, USA

\section{Introduction}

Congenital thrombotic thrombocytopenic purpura (cTTP) is a rare autosomal recessive disorder resulting from ADAMTS13 gene mutations that lead to severe deficiency of this metalloproteinase [1]. Decreased ADAMTS13 activity allows the circulation of ultra-large von Willebrand factor multimers and increases the risk of microvascular thrombosis [2]. The brain vasculature is particularly susceptible to TTP-associated thrombosis and is a common clinical manifestation of congenital ADAMTS13 deficiency [3, 4]. We describe a middle-aged patient with history of recurrent cryptogenic strokes in whom the diagnosis of cTTP was made, highlighting this disorder in the differential diagnosis of idiopathic strokes and the role of recombinant ADAMTS13 in the prevention of recurrent events.

\section{Case description}

A 58-year-old female presented to her primary care physician in August 2020 for lingual bleeding and right upper arm ecchymoses. A complete blood count revealed platelet count of 38,000 cells $/ \mathrm{mm}^{3}$ and the patient was referred to a 
hematologist. She had a history of abnormal bruising as a teenager, on the thighs and posterior side of upper arms. She reported at least two instances of having thrombocytopenia in the 1990s, following general anesthesia for myomectomy and in $2002\left(142,000\right.$ cells $\left./ \mathrm{mm}^{3}\right)$. Mild thrombocytopenia was noted in 2017 during a routine visit to primary care physician $\left(109,000\right.$ cells $\left./ \mathrm{mm}^{3}\right)$. Her obstetric history included two spontaneous first trimester abortions. Past medical history was remarkable for an ischemic stroke at age 39 after hormonal treatment (leuprolide and estrogen) for ovarian stimulation for in-vitro fertilization, and a transient ischemic attack (TIA) in 2002. She had been on chronic anticoagulation with warfarin since 2002. Thrombophilia evaluation in 2006 showed a mildly decrease protein $\mathrm{S}$ activity $55 \%$ (range $62-163 \%$ ), protein $\mathrm{S}$ antigen free $60 \%$ (range $70-160 \%$ ) and a normal protein S antigen total 104\% (range 70-175\%) while off warfarin. In April 2020 she was admitted with left leg weakness after an episode of fever, chills, cough and myalgias post COVID-19 exposure 3 weeks prior. SARSCoV-2 RT-PCR was negative. A brain magnetic resonance imaging (MRI) revealed a right superior frontal gyrus acute infarct and a right inferior frontal gyrus subacute infarct. The international normalized ratio (INR) on admission was 1.8. Anticoagulation was switched to apixaban $5 \mathrm{mg}$ twice daily. Her platelet count was 172,000 cells $/ \mathrm{mm}^{3}$ on admission but decreased to 131,000 cells $/ \mathrm{mm}^{3}$ at the time of discharge. At the time of Hematology consultation in August 2020, the patient had no neurologic symptoms, but physical examination revealed an ecchymosis on the posterior aspect of the right arm (Fig. 1A) and lingual ecchymosis with ulceration (Fig. 1B). Her medications included apixaban, albuterol, levothyroxine, and atorvastatin. Laboratory tests showed a hemoglobin $(\mathrm{Hb})$ of $11.4 \mathrm{~g} / \mathrm{dl}$, platelets 55,000 cells $/ \mathrm{mm}$ [3], reticulocytes $2.37 \%$, LDH $624 \mathrm{mg} / \mathrm{dl}$, and haptoglobin $92 \mathrm{mg} / \mathrm{dl}$. Blood film revealed five schistocytes/high power field (Fig. 1C) and decreased platelets. She was positive for SARS-CoV-2 antibodies. She was hospitalized with presumptive diagnosis of TTP. Prior to the initiation of plasma infusion, she developed slurred speech, left facial palsy and right gaze preference. Brain MRI demonstrated early sub-acute infarction in the right frontal lobe (Fig. 1D) and multi-focal regions of encephalomalacia and gliosis compatible with chronic infarctions. She was started on plasma exchange (PLEX). Pre-treatment ADAMTS13 activity was $<5 \%$ and an inhibitor was not detected.

After four PLEX treatments, ADAMTS13 activity increased to $9 \%$, her lingual lesion resolved, and the platelet count normalized. ADAMTS13 gene sequencing revealed a homozygous $3070 \mathrm{~T}>\mathrm{G}$ mutation in exon 24 leading to the diagnosis of cTTP. Maintenance treatment with $10 \mathrm{ml} / \mathrm{kg}$ of plasma failed to maintain ADAMTS13 activity $\geq 10 \%$. In May 2021 she was hospitalized with new onset of disorientation and right facial droop. Brain MRI demonstrated an acute left thalamic infarct. This episode occurred after a proteus mirabilis urinary tract infection. On admission, the ADAMTS13 activity was undetectable without evidence of an inhibitor. She was treated initially with daily plasma and ADAMTS 13 activity increased to 35\%. Due to the recurrent stroke and failure to maintain ADAMTS13 activity level $>10 \%$ while on plasma, recombinant ADAMTS 13 (TAK-755) was obtained through compassionate use and an emergent investigational application to the FDA. The patient received a loading dose of $40 \mathrm{U} / \mathrm{kg}$ on day $1,20 \mathrm{U} / \mathrm{kg}$ on day 2 , and $15 \mathrm{U} / \mathrm{kg}$ on days 3 through 6 , achieving peak ADAMTS 13 activity $>90 \%$ and trough $>60 \%$ during the loading period. She then received $40 \mathrm{U} / \mathrm{kg}$ weekly, achieving ADAMTS13 activity trough levels between 16 and $21 \%$ with no further recurrences, normalization of the platelet count and with minimal neurologic sequelae. She was readmitted 2 weeks after the last stroke with vaginal bleeding in the setting of uterine fibroids. Anticoagulation was discontinued and she is currently on aspirin $81 \mathrm{mg}$ daily.

The patient's family ancestry is from the island of Montserrat. Her parents had no thrombotic history but one of her two sisters had a TIA and a deep vein thrombosis and is heterozygote for the $3070 \mathrm{~T}>\mathrm{G}$ mutation with an ADAMTS13 activity of $48 \%$.

\section{Discussion}

ADAMTS13 exon 24 mutations are associated with late presentation of cTTP, often triggered by pregnancy [3]. In addition to the specific mutation and residual protein function, other factors influence the occurrence of thrombotic microangiopathy (TMA) in patients with cTTP. Those include states associated with high von Willebrand factor levels, such as pregnancy [4]. In our patient, surgery/general anesthesia, hormonal treatment, and infections have been the triggers of TMA and strokes. In the UK TTP registry, half of patients with congenital TTP had had prior clinical manifestations of the disease before acute events leading to the diagnosis [3]. In that cohort, delayed recognition of cTTP was associated with worse outcomes.

Strokes/TIAs affect over half of patients with delayed recognition of cTTP, imposing serious morbidity [3]. The importance of ADAMTS13 activity in stroke has been recognized in older adults in the general population. In one study, ADAMTS13 activity $<80 \%$ was associated with higher incidence of stroke independent from cardiovascular risk factors [5]. Low ADAMTS13 activity in relation to von Willebrand factor antigen concentrations at presentation for ischemic stroke were associated with worse neurologic deficits and increased mortality [6]. Despite these reports, TTP (congenital or acquired) is rarely considered in the differential diagnosis of cryptogenic strokes [7]. 


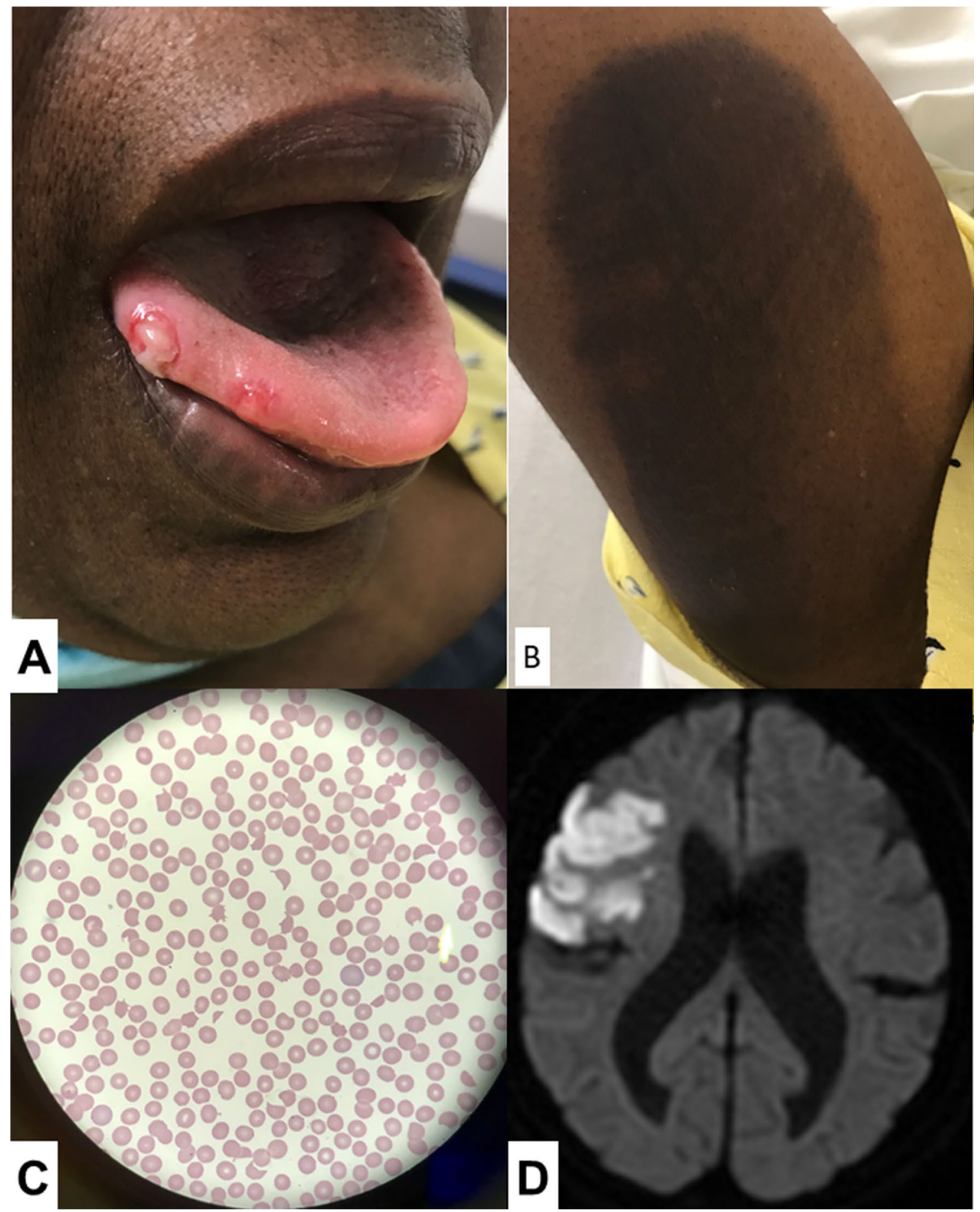

Fig. 1 Panel A. Lingual lesion; Panel B. Arm ecchymoses; Panel C. Blood film showing schistocytes, occasional polychromasia and decreased platelets; Panel D. Magnetic Resonance Imaging of the

As exemplified by our patient's history, cTTP is likely to be under-recognized as the cause of recurrent cryptogenic strokes, given the rarity of this condition and potentially subtle hematologic findings. However, prompt brain demonstrating subacute infarction in the right frontal lobe in the right middle cerebral artery territory

recognition of cTTP offers opportunities to decrease morbidity and mortality in these patients. Data from patients with immune TTP who survive the initial presentation suggest that ADAMTS13 level $<70 \%$ after the recovery 
from an acute TTP episode is associated with an almost tenfold higher risk of stroke when compared to age- and sex-matched controls [8]. Consistent with that, prophylactic ADAMTS13 infusions are thought to decrease strokes in patients with cTTP based on non-randomized studies [3].

Plasma is commonly used as source of ADAMTS13, and alternatives used mostly in Europe include intermediate-purity factor VIII concentrate [3]. However, plasma may be insufficient as illustrated by our patient's recurrent stroke and ADAMTS13 level of 0\% during the acute event, and as documented in a Japanese cohort [9]. Taylor et al. [10] investigated the pharmacokinetic of ADAMTS13 in patients in cTTP, deriving two important observations. First, there was a wide variation in ADAMTS13 clearance (3.4-7.9 days). Second, ADAMTS13 trough levels $>10 \mathrm{IU} / \mathrm{dl}$ were achievable with individualized FFP administration protocols, however plasma trough levels $>50 \mathrm{IU} / \mathrm{dl}$ were unfeasible due to the risk of volume overload. This limitation to plasma therapy may leave patients with excessive risk of strokes.

Plaimauer et al. [11] reported the cloning of human ADAMTS13 gene in 2002, and the same group reported treating two brothers affected by cTTP with the recombinant form of ADAMTS13 (rADAMTS13) in 2003 [12]. rADAMTS13 was also used to bypass auto-antibodies in a patient with acquired TTP [13]. Scully et al. [14] reported the first-in-human pharmacokinetics and safety study of BAX 930 in 2017, with no infusion reactions or development of anti-rADAMTS13 antibodies noted. Now two agentsBAX 930 and TAK-755-are under study in phase three clinical trials in patients with cTTP. The primary endpoint in the study with BAX930 is the number of participants with acute TTP episodes, for TAK-755 the main endpoint is treatment safety. Both studies are assessing neurological symptoms as a secondary outcome (ClinicalTrials.gov Identifier: NCT03393975 and NCT04683003).

Early recognition and expanded access to recombinant ADAMTS13 and other novel therapies through clinical trials are pivotal to improve outcomes in patients with cTTP [4]. The clinical utility of these novel therapies needs to be systematically assessed in the management and prevention of acute TTP episodes, and for the long-term, insidious consequences of ADAMTS13 deficiency, such as obstetric morbidity and strokes. Our patient's case illustrates both the challenges to recognize and diagnose cTTP and the promises that rADAMTS13 holds in the chronic management of this condition.

Supplementary Information The online version contains supplementary material available at https://doi.org/10.1007/s11239-021-02629-7.
Acknowledgements We acknowledge the assistance of Dr. Nisha Jain (Takeda Pharmaceuticals) in securing the recombinant ADAMTS13 for our patient and Dr. Alan Segal Neurology attending at Weill Cornell Medical Center as the patient's primary neurologist.

Author contributions MBM collected the data and drafted the initial manuscript. MTD conceived the idea for writing this case report, collected the data, wrote the manuscript, and did the final approval of the manuscript.

\section{Declarations}

Conflict of interest There are no potential conflicts of interest for any of the authors in reference to this case report.

\section{References}

1. Kokame K, Matsumoto M, Soejima K et al (2002) Mutations and common polymorphisms in ADAMTS13 gene responsible for von Willebrand factor-cleaving protease activity. Proc Natl Acad Sci USA 99(18):11902-11907

2. Remuzzi G, Galbusera M, Noris M et al (2002) von Willebrand factor cleaving protease (ADAMTS13) is deficient in recurrent and familial thrombotic thrombocytopenic purpura and hemolytic uremic syndrome. Blood 100(3):778-785

3. Alwan F, Vendramin C, Liesner R et al (2019) Characterization and treatment of congenital thrombotic thrombocytopenic purpura. Blood 133(15):1644-1651

4. Kremer Hovinga JA, George JN (2019) Hereditary thrombotic thrombocytopenic purpura. N Engl J Med 381(17):1653-1662

5. Sonneveld MA, de Maat MP, Portegies ML et al (2015) Low ADAMTS13 activity is associated with an increased risk of ischemic stroke. Blood 126(25):2739-2746. https://doi.org/10.1182/ blood-2015-05-643338

6. Taylor A, Vendramin C, Singh D, Brown MM, Scully M (2020) von Willebrand factor/ADAMTS13 ratio at presentation of acute ischemic brain injury is predictive of outcome. Blood Adv 4(2):398-407

7. McMahon NE, Bangee M, Benedetto V et al (2020) Etiologic workup in cases of cryptogenic stroke. Stroke 51(5):1419-1427

8. Upreti H, Kasmani J, Dane K et al (2019) Reduced ADAMTS13 activity during TTP remission is associated with stroke in TTP survivors. Blood 134(13):1037-1045

9. Sakai K, Fujimura Y, Miyata T, Isonishi A, Kokame K, Matsumoto M (2021) Current prophylactic plasma infusion protocols do not adequately prevent long-term cumulative organ damage in the Japanese congenital thrombotic thrombocytopenic purpura cohort. $\mathrm{Br} \mathbf{J}$ Haematol 194(2):444-452. https://doi.org/10.1111/bjh.17560

10. Taylor A, Vendramin C, Oosterholt S, Della Pasqua O, Scully M (2019) Pharmacokinetics of plasma infusion in congenital thrombotic thrombocytopenic purpura. J Thromb Haemost 17(1):88-98. https://doi.org/10.1111/jth.14345

11. Plaimauer B, Zimmermann K, Volkel D et al (2002) Cloning, expression, and functional characterization of the von Willebrand factor-cleaving protease (ADAMTS13). Blood 100(10):3626-3632

12. Antoine G, Zimmermann K, Plaimauer B et al (2003) ADAMTS13 gene defects in two brothers with constitutional thrombotic thrombocytopenic purpura and normalization of von Willebrand factorcleaving protease activity by recombinant human ADAMTS13. Br J Haematol 120(5):821-824. https://doi.org/10.1046/j.1365-2141. 2003.04183.x 
13. Plaimauer B, Kremer Hovinga JA, Juno C et al (2011) Recombinant ADAMTS13 normalizes von Willebrand factor-cleaving activity in plasma of acquired TTP patients by overriding inhibitory antibodies. J Thromb Haemost 9(5):936-944. https://doi.org/10.1111/j.15387836.2011.04224.x

14. Scully M, Knobl P, Kentouche K et al (2017) Recombinant ADAMTS-13: first-in-human pharmacokinetics and safety in congenital thrombotic thrombocytopenic purpura. Blood 130(19):2055-2063

Publisher's Note Springer Nature remains neutral with regard to jurisdictional claims in published maps and institutional affiliations. 